\title{
Propriedades Psicométricas do Inventário de Funcionamento Psicológico Positivo: Versões Breve e Abrangente
}

\author{
Larissa Maria David Gabardo-Martins*, 1 \\ Orcid.org/0000-0003-1356-8087 \\ Maria Cristina Ferreira ${ }^{1}$ \\ Orcid.org/0000-0003-0752-6710
}

${ }^{1}$ Universidade Salgado de Oliveira, Niterói, RJ, Brasil

\begin{abstract}
Resumo
O bem-estar é um dos construtos mais estudados no âmbito da Psicologia Positiva. Um indicador recente do referido construto é o funcionamento psicológico positivo, que se relaciona a um estado positivo de funcionamento mental, físico e social. O objetivo do estudo foi reunir evidências de validade das versões abrangente e breve do Inventário de Funcionamento Psicológico Positivo, em amostras brasileiras. O estudo contou com 801 participantes, de ambos os sexos, com idades variando entre 16 e 76 anos, que responderam à versão brasileira das escalas e a outros instrumentos. As análises fatoriais confirmatórias demonstraram que a versão abrangente ficou composta por 54 itens, distribuídos igualmente em 18 fatores de primeira ordem, sendo que a versão breve ficou composta por 10 itens distribuídos em um único fator. Na validação convergente, os fatores positivos da versão abrangente e da versão breve correlacionaram-se positivamente com a satisfação com a vida, o otimismo, as avaliações autorreferentes e o florescimento, enquanto os fatores negativos da versão abrangente correlacionaram-se negativamente com esses construtos. De acordo com os resultados, recomenda-se o uso das escalas em investigações futuras sobre o funcionamento psicológico positivo.
\end{abstract}

Palavras-chave: Bem-estar, funcionamento psicológico positivo, validação de instrumentos, Psicometria.

\section{Psychometric Properties of the Inventory of Thriving: Brief and Comprehensive Versions}

\begin{abstract}
Well-being is one of the most studied constructs in the field of Positive Psychology. A recent indicator of this construct is the thriving, which is related to a positive state of mental, physical and social functioning. The present study's objective was to collect validity evidence for the comprehensive and brief versions of the Inventory of Thriving in Brazilian samples. The study involved 801 participants, of both sexes, between the ages of 16 and 76 years, who responded to the Brazilian versions of the scales and other

* Endereço para correspondência: Universidade Salgado de Oliveira, R. Marechal Deodoro, 211, Bloco C, Centro, Niterói, RJ, Brasil 24030-060. Fone: (021) 2138-4926; Fax: (021) 2138-4926. E-mail: gabardolarissa@) gmail.com e mcris@centroin.com.br
\end{abstract}


instruments. Confirmatory factor analysis showed that the comprehensive version was composed of 54 items equally distributed among 18 first-order factors; and the brief version was composed of a single factor consisting of 10 items. With respect to convergent validity, the comprehensive and brief versions' positive factors were positively correlated with life satisfaction, optimism, core self-evaluation, and flourishing, while the comprehensive version's negative factors were negatively correlated with these constructs. According to the results, it is recommended to use the scales in future research on thriving.

Keywords: Well-being, thriving, validation of instruments, Psychometrics.

\section{Propiedades Psicométricas del Inventario de Funcionamiento Psicológico Positivo: Versiones Breve y Comprensiva}

\section{Resumen}

El bienestar es uno de los constructos más estudiados en el ámbito de la Psicología Positiva. Un indicador reciente de dicho constructo es el funcionamiento psicológico positivo, que se relaciona con un estado positivo de funcionamiento mental, físico y social. El objetivo del estudio era reunir pruebas de validez de las versiones comprensiva y breve de el Inventario de Funcionamiento Psicológico Positivo en muestras brasileñas. El estudio incluyó a 801 participantes, de ambos sexos, con edades comprendidas entre los 16 y los 76 años, que respondieron a la versión brasileña de las escalas y otros instrumentos. El análisis factorial confirmatorio mostró que la versión comprensiva se compone de 54 itens, igualmente distribuidos en 18 factores de primer orden, y la versión breve se compone de 10 itens distribuidos en un solo factor. En validez convergente, los factores positivos de la versión comprensiva y la versión breve se correlacionarón positivamente con la satisfacción con la vida, optimismo, evaluaciones autorreferenciales y floración, mientras que los factores negativos de la versión comprensiva una correlación negativa con estes construtos. De acuerdo con los resultados, se recomienda el uso de las escalas en investigaciones futuras sobre el funcionamiento psicológico positivo.

Palabras clave: Bienestar, funcionamiento psicológico positivo, validación de instrumentos, Psicometría.

Ao final da Segunda Guerra Mundial, a Psicologia passou a adotar uma perspectiva eminentemente curativa, associada à reparação dos danos causados pelas doenças (Seligman, 2004) e em tudo aquilo que não estava funcionando bem no indivíduo (Seligman \& Csikszentmihalyi, 2000). Em reação a este movimento, surge, ao final do século XX, a Psicologia Positiva, que passou a promover pesquisas focalizadas nas forças, no potencial das pessoas (Snyder \& Lopez, 2009) e no desenvolvimento do que há de melhor nos indivíduos, grupos e instituições (Seligman \& Csikszentmilahly, 2000).

Um dos construtos que adquiriu maior visibilidade nessa nova perspectiva é o bem-estar, que vem sendo abordado por meio de duas principais correntes: a hedônica e a eudaimônica. A primeira concentra-se no bem-estar subjeti- vo, considerado como sinônimo de felicidade ou prazer (Huta \& Ryan, 2010). O bem-estar subjetivo associa-se, portanto, à vivência de emoções agradáveis, de baixos níveis de humor negativo e de elevada satisfação com a vida (Diener, Oishi, \& Lucas, 2002). A corrente eudaimônica, por sua vez, dedica-se ao estudo do bem-estar psicológico, defendendo que ele vai além da felicidade, por estar associado à autorrealização. Nesse sentido, Ryan e Deci (2008) defendem que o bem-estar psicológico ultrapassa o simples relato sobre a felicidade, porque as emoções positivas ou negativas não são capazes, por si só, de garantir o equilíbrio psicológico e a realização plena do potencial humano.

Mais recentemente, porém, tem se tornado mais comum a integração das perspectivas he- 
dônica e eudaimônica, ao invés de sua separação em duas abordagens distintas. Um exemplo de tal integração é o modelo de bem-estar proposto por Seligman (2011), o PERMA (Positive emotions, Engagement, Relationships, Meaning, Accomplislishments), segundo o qual há cinco caminhos para o alcance do bem-estar: o prazer (emoções positivas), o engajamento, o relacionamento positivo, o significado e a realização.

Outra proposta de integração entre essas duas correntes é a de Su, Tay e Diener (2014). Adotando o termo thriving (prosperidade), os autores caracterizam o referido construto como um estado de funcionamento mental, físico e social positivo de natureza multidimensional, composto por sete dimensões teóricas centrais. São elas: o bem-estar subjetivo; as relações de apoio; o interesse e o engajamento em atividades diárias; o significado e o propósito na vida; o senso de domínio e realização; os sentimentos de controle e autonomia; e o otimismo ( $\mathrm{Su}$ et al., 2014). Estas sete dimensões subdividem-se, ainda, em 18 fatores: satisfação com a vida, afetos positivos e negativos (dimensão bem-estar subjetivo); suporte social, comunidade, confiança, respeito, solidão, pertencimento (dimensão relações de apoio); engajamento (dimensão interesse e engajamento em atividades diárias); significado (dimensão significado e propósito na vida); habilidades, aprendizagem, realização, autoeficácia e autoestima (dimensão senso de domínio e realização); controle (dimensão sentimentos de controle e autonomia); otimismo (dimensão otimismo). Segundo Su et al. (2014), todos esses aspectos revelam-se essenciais para uma vida saudável, longa, completa e feliz. Cumpre ressaltar que, no presente trabalho, a expressão funcionamento psicológico positivo foi adotada para nomear o instrumento, em função de considerarmos que a tradução literal do termo thriving por prosperidade não daria a conotação que os autores dão ao termo, qual seja a de um estado de funcionamento mental positivo de natureza multidimensional. Em outras palavras, $\mathrm{Su}$ et al. (2014) afirmam várias vezes, ao longo de seu texto, que o foco do instrumento é o funcionamento psicológico positivo.
No que se refere à avaliação das manifestações do bem-estar sob a perspectiva hedônica, merecem destaque a Escala de Satisfação com a Vida (Diener, Emmons, Larsen, \& Griffin, 1985) e a Escala de Experiências Positivas e Negativas (Diener et al., 2009). A Escala de Satisfação com Vida foi desenvolvida e apresentou evidências iniciais de validade nos Estados Unidos, em estudantes universitários. A análise fatorial exploratória evidenciou uma estrutura de um fator com cinco itens, enquanto a análise de correlação demonstrou que o instrumento relaciona-se com outras medidas de bem-estar subjetivo. O referido instrumento obteve evidências de validade para o contexto brasileiro no estudo de Gouveia, Barbosa, Andrade e Carneiro (2005), em uma amostra de médicos, no qual as análises fatoriais exploratória e confirmatória indicaram que a estrutura unifatorial também se manteve.

A Escala de Experiências Positivas e Negativas foi desenvolvida por Diener et al. (2009), em cinco diferentes amostras dos Estados Unidos e apresentou evidências iniciais de validade. As análises fatoriais exploratórias evidenciaram uma estrutura de dois fatores (experiências positivas e negativas), cada um com seis itens. Tal instrumento ainda não foi adaptado para o contexto brasileiro.

Existem, ainda, alguns instrumentos desenvolvidos no Brasil, com o objetivo de avaliar o bem-estar subjetivo, como é o caso, por exemplo, da Escala de Bem-Estar Subjetivo de Albuquerque e Troccóli (2004), desenvolvida e validada em um estudo com policiais civis brasileiros. Os resultados obtidos nas análises fatoriais exploratórias deram origem a uma estrutura final de 62 itens, divididos em três fatores: afetos positivos, afetos negativos e satisfação com a vida.

Outra escala desenvolvida no contexto brasileiro é a Escala de Afetos de Zanon, Bastianello, Pacico e Hutz (2013). O estudo de desenvolvimento do referido instrumento contou com a participação de 853 universitários do sul do país. A análise fatorial exploratória demonstrou que a escala ficou composta por 20 itens, distribuídos igualmente em dois fatores: afetos positivos e afetos negativos. 
Sob a perspectiva eudaimônica, um instrumento frequentemente utilizado para avaliar o bem-estar é a Escala de Bem-Estar Psicológico (Ryff, 1989). O instrumento original é composto de 120 itens, distribuídos por seis fatores (cada um com 20 itens) e obteve evidências iniciais de validade em uma amostra norte-americana. As análises fatoriais exploratórias evidenciaram uma estrutura de seis fatores (autoaceitação, relação positiva com os outros, crescimento pessoal, propósito na vida, domínio ambiental e autonomia) correlacionados entre si e com outros construtos do bem-estar (Ryff, 1989). Posteriormente, versões alternativas e mais breves do referido instrumento foram desenvolvidas, como a versão de Ryff e Keyes (1995), com 18 itens e três dimensões, e a versão de Ryff e Essex (1992), com 84 itens (14 por fator). Esta última obteve evidências de validade para o contexto brasileiro em investigação de Machado, Bandeira e Pawlowski (2013), com 313 estudantes universitários. A validade fatorial foi avaliada por meio de análises fatoriais confirmatórias e a validade convergente, por análises de correlação com indicadores de satisfação com a vida, afetos positivos e negativos, balanço entre afetos e depressão. Os resultados evidenciaram que modelo de seis fatores foi o que apresentou melhor ajuste, sendo que as seis dimensões se correlacionaram conforme esperado com os indicadores de bem-estar.

Mais recentemente, porém, tem se tornado também comum a integração das perspectivas hedônica e eudaimônica na mensuração do bem-estar. Sob essa ótica, foi desenvolvida a Escala de Florescimento (Diener et al., 2010), em estudo com uma amostra de 689 estudantes estadunidenses. A análise fatorial exploratória resultou em uma escala de estrutura unifatorial, composta por 8 itens. $\mathrm{O}$ referido instrumento ainda não teve suas características psicométricas testadas em amostras brasileiras. Contudo, ela foi adaptada para o contexto do trabalho por Mendonça, Caetano, Ferreira, Sousa e Silva (2014), em uma amostra brasileira.

As medidas até aqui desenvolvidas para a avaliação do bem-estar têm abordado somente algumas das manifestações desse construto.
Acrescente-se a isso o fato de que, no contexto da área médica, o foco das avaliações ainda é a doença, isto é, o mal-estar (Su et al., 2014). Procurando preencher essas lacunas, especialmente no que diz respeito à promoção de uma abordagem mais positiva na área da saúde, Su et al. (2014) recentemente desenvolveram e reuniram evidências de validade, nos Estados Unidos, de uma medida mais abrangente de bem-estar.

$\mathrm{O}$ instrumento tem como objetivo mensurar os 18 fatores que compõem o modelo de bem-estar de Su et al. (2014) e foi denominado de "Comprehensive Inventory of Thriving" ("Inventário Abrangente de Funcionamento Psicológico Positivo" - IAFPP). Os autores desenvolveram, ainda, uma versão breve da escala ("Inventário Breve de Funcionamento Psicológico Positivo" - IBFPP), que se compõe de dez itens e é considerada uma medida central do bem-estar ( $\mathrm{Su}$ et al., 2014).

No estudo original de desenvolvimento e validação de ambos os instrumentos, participaram 3191 indivíduos estadunidenses (Su et al., 2014), divididos em cinco diferentes amostras:(a) 490 estudantes de graduação; (b) 551 idosos; (c) 501 indivíduos com baixos níveis socioeconômicos; (d) 559 adultos e (e) 1090 adultos. A análise fatorial confirmatória multigrupos das quatro amostras revelou que a estrutura de 18 fatores correlacionados, cada um com três itens, mostrou-se consistente entre os grupos, e que os fatores latentes eram correlacionados uns com os outros (Su et al., 2014). Tais fatores distribuíam-se em 15 positivos (suporte, comunidade, confiança, respeito, pertencimento, engajamento, habilidades, aprendizagem, realização, autoeficácia, autoestima, significado, otimismo, satisfação com a vida e afetos positivos) e três negativos (solidão, controle e afetos negativos). Os índices de confiabilidade dos 18 fatores, calculado pelo Alfa de Cronbach, variaram de 0,71 a 0,96, nas diferentes amostras.

A partir das médias das cargas fatoriais dos itens dos fatores latentes, foram selecionados os dez itens para a versão breve do instrumento. Em seguida, a estrutura da versão breve foi testada por meio da análise fatorial exploratória, tendo obtido evidências para a estrutura de um 
fator, que explicou $57,53 \%$ da variância total dos itens. As cargas fatoriais dos itens nesta versão variaram de 0,58 a 0,84 (Su et al., 2014). A média dos índices de confiabilidade nas diferentes amostras, calculadas pelo Alfa de Cronbach, foi de 0,90 nessa versão breve.

Com a finalidade de obter evidências sobre a validade convergente dos instrumentos, foram aplicados na amostra quatro outros instrumentos correlatos ao bem-estar (satisfação com a vida, florescimento, controle, otimismo e avaliações autorreferentes), sendo que as análises de correlação demonstraram que os fatores do IAFPP e o IBFPP correlacionavam-se com tais construtos, o que atestou a validade convergente dos dois instrumentos (Su et al., 2014). No que diz respeito à validade preditiva, as análises de regressão indicaram que as dimensões do IAFPP e o IBFPP predisseram significativamente quatro resultados de saúde: estado geral de saúde relacionado à qualidade de vida; presença de condições médicas (como pressão alta); nível de funcionamento físico; comportamentos de saúde (como beber suco de fruta ou praticar exercícios físicos).

Com o intuito de obter a confiabilidade teste-reteste dos instrumentos, os indivíduos da amostra cinco responderam ao instrumento em dois tempos, com espaço de quatro meses entre uma e outra aplicação. A confiabilidade teste-reteste para o IBFPP foi de 0,83 , tendo variado de 0,57 a 0,83 para as dimensões do IAFPP ( $\mathrm{Su}$ et al., 2014), com apenas o fator afetos negativos tendo apresentado um índice menor que 0,60.

A versão breve do Inventário de Funcionamento Psicológico Positivo obteve também evidências de validade no contexto chinês, em investigação de Duan, Guan e Gan (2016), realizada com 705 chineses da comunidade geral e 251 estudantes universitários. Os resultados obtidos confirmaram a estrutura de um fator e reuniram evidências de validade convergente $\mathrm{e}$ discriminante com outras medidas de bem-estar (Escala de Satisfação com Vida, Escala de Florescimento, Questionário de Sentido de Vida, Escala de Afetos Positivos e Negativos). Além disso, eles demonstraram que o funcionamento psicológico positivo prediz resultados de saúde mental, mensurados por escalas de depressão, ansiedade e estresse.

Até o momento, todos os instrumentos de mensuração do bem-estar com evidências de validade para o contexto brasileiro têm abordado somente algumas de suas manifestações, conforme levantamento realizado em bases de dados nacionais (Scientific Electronic Library Online [SciELO] e Pepsic), em dezembro de 2016. Ademais, a consulta às bases de dados PsycInfo, American Psychiatric Association (APA), SciELO e Pepsic, realizada em dezembro de 2016, com as palavras-chave "Brief Inventory of Thriving", "Comprehensive Inventory of Thriving" $\mathrm{e}$ "scale development", revelou que o único estudo que apresenta evidências de validade da versão abrangente do instrumento é o de $\mathrm{Su}$ et al. (2014), realizado nos Estados Unidos, sendo que a versão breve do instrumento obteve evidências de validade apenas nos contextos estadunidense e chinês. Outrossim, as medidas para a área da saúde no Brasil ainda se focam, sobretudo, nas doenças, isto é, o contexto brasileiro carece de medidas com abordagem mais positiva na área da saúde (Coluci, Alexandre, \& Milani, 2015). Considerando, porém, que o bem-estar é um importante componente da saúde, que auxilia na melhora da saúde física e na emissão de comportamentos saudáveis ( $\mathrm{Su}$ et al., 2014), tornam-se necessários mais estudos brasileiros que contribuam para o preenchimento de tais lacunas.

Fundamentando-se em tais considerações, o presente estudo teve como objetivo geral reunir evidências iniciais de validade de estrutura, de consistência interna e convergente do IAFPP em amostras brasileiras. Tais instrumentos poderão mostrar-se úteis aos estudiosos do bem-estar e da saúde mental, em função de se constituírem em medidas mais abrangentes que incluem uma variedade de manifestações do bem-estar. Além disso, o IAFPP poderá auxiliar no desenvolvimento de intervenções para a melhoria do bem-estar, ao oferecer um diagnóstico inicial sobre a saúde mental dos indivíduos, que destaca seus pontos fortes e as áreas que precisam ser melhoradas.

Para a realização da validade convergente do instrumento, foram usados outros construtos 
correlatos ao bem-estar, quais sejam: satisfação com a vida, otimismo, avaliações autorreferentes, florescimento e saúde geral. A satisfação com a vida refere-se a um processo de julgamento e avaliação positiva geral da própria vida, que depende da comparação entre as circunstâncias da vida do indivíduo a um padrão por ele previamente definido (Emmons, 1986). O otimismo diz respeito à atribuição de explicações permanentes, difundidas e internas para os eventos bons, e explicações temporárias, específicas e externas para os eventos ruins (Seligman, 1995).

As avaliações autorreferentes relacionam-se a premissas fundamentais que os indivíduos apresentam acerca de si mesmos, as quais se compõem de quatro traços: autoestima, autoeficácia generalizada, estabilidade emocional (Judge, Locke, \& Durham, 1997) e lócus de controle (Judge \& Bono, 2001). O florescimento diz respeito ao bom funcionamento do indivíduo nos aspectos psicológicos e sociais (Keyes, 2002).

Considerando-se, por fim, que o bem-estar vem sendo associado a vários resultados de saúde (Su et al., 2014), a saúde geral também foi utilizada para a verificação da validade convergente dos instrumentos. Tal construto relaciona-se aos desvios comportamentais de um indivíduo, que pode apresentar ou não doenças psiquiátricas não severas (não psicóticas) (Sarriera, Schwarcz, \& Câmara, 1996). Partindo-se do pressuposto de que os construtos utilizados na validação convergente caracterizam-se como estados positivos dos indivíduos, e que os fatores do IAFPP caracterizam-se como estados positivos ou negativos, foram formuladas as seguintes hipóteses:

Hipótese 1: Os fatores positivos do IAFPP e o IBFPP apresentam correlações positivas de moderadas a fortes com a satisfação com a vida, o otimismo, as avaliações autorreferentes, o florescimento e a saúde geral.

Hipótese 2: Os fatores negativos do IAFPP apresentam correlações negativas de moderadas a fortes com a satisfação com a vida, o otimismo, as avaliações autorreferentes, o florescimento e a saúde geral.

\section{Método}

\section{Participantes}

A amostra foi composta por 801 participantes, de ambos os sexos, provenientes de 24 estados brasileiros, mais o Distrito Federal, com destaque para os estados de São Paulo (28,7\%), Rio de Janeiro (16,5\%) e Minas Gerais (12,7\%). Do total de respondentes, a maioria era de mulheres $(59,8 \%)$. Quanto ao estado civil, grande parte era solteira (48,4\%), seguida de casada $(39,7 \%)$. No que diz respeito à escolaridade, $30,3 \%$ indicaram mestrado completo, $27,3 \%$, ensino médio completo e $25,2 \%$ graduação completa. A idade da amostra variou de 16 a 76 anos $(M=33,06 ; D P$ $=9,40)$ e o tempo de serviço, de 1 a 48 anos $(M$ $=6,78 ; D P=8,73)$. Em relação ao fato de a pessoa estar trabalhando ou não, 598 participantes $(74,7 \%)$ declararam que estavam trabalhando. $\mathrm{O}$ único critério de inclusão utilizado foi que os respondentes deveriam ter, pelo menos, ensino médio completo, para a melhor compreensão dos itens dos instrumentos.

\section{Instrumentos}

O funcionamento psicológico positivo foi mensurado pelo Inventário Abrangente de Funcionamento Psicológico Positivo, desenvolvido por Su et al. (2014), o qual se compõe de 54 itens, divididos igualmente em 18 fatores, a serem respondidos por meio de escalas do tipo Likert de cinco pontos (1 - Discordo fortemente a 5 - Concordo fortemente). Do total dos fatores, 15 são positivos (suporte social, comunidade, confiança, respeito, pertencimento, engajamento, habilidades, aprendizagem, realização, autoeficácia, autoestima, significado, otimismo, satisfação com a vida e afetos positivos) e três são negativos (solidão, controle e afetos negativos). Exemplo de item positivo: "Minha vida tem um claro sentido de propósito". Exemplo de item negativo: "Outras pessoas tomam a maioria das decisões da minha vida". Para a tradução da escala, foi adotado o procedimento de tradução e retradução (back-translation), que consiste em uma tradução dos itens para o português, seguida da tradução desta versão novamente para o 
inglês e da comparação dessas duas versões, a fim de verificar se houve uma equivalência conceitual (Borsa, Damásio, \& Bandeira, 2012). Seguindo algumas das recomendações desses autores, foram realizadas três etapas: (1) tradução do instrumento original do idioma inglês para o português, por um pesquisador fluente em inglês; (2) tradução da versão em português para o inglês, por um professor de língua inglesa; (3) revisão técnica e avaliação semântica das duas versões em inglês por dois pesquisadores da área de psicometria.

A satisfação com a vida foi medida pela Escala de Satisfação com a Vida de Diener et al. (1985), adaptada para amostras brasileiras por Gouveia et al. (2003). O instrumento é unifatorial e composto de cinco itens, a serem respondidos por meio de escala do tipo Likert de sete pontos (1 - Discordo totalmente a 7 - Concordo totalmente). Exemplo de item do instrumento: "Estou satisfeito(a) com minha vida". Na adaptação para o contexto brasileiro (Gouveia et al., 2003), o índice de consistência interna, avaliado pelo Alfa de Cronbach, foi de 0,72 e na, presente investigação, foi de 0,88 .

O otimismo foi medido pelo Teste de Orientação da Vida, construído por Scheier e Carver (1985), e adaptado ao contexto brasileiro por Bandeira, Bekou, Lott, Teixeira e Rocha (2002). $\mathrm{O}$ instrumento é unifatorial e composto de sete itens, a serem respondidos em escala do tipo Likert de cinco pontos (1 - Discordo totalmente a 5 - Concordo totalmente). Exemplo de item: "Nos momentos de incerteza, geralmente eu espero que aconteça o melhor". Na adaptação ao contexto brasileiro (Bandeira et al., 2002), o índice de consistência interna, calculado pelo Alfa de Cronbach, foi de 0,68 , e de 0,71 , na presente investigação.

As avaliações autorreferentes foram mensuradas pela escala de Judge, Erez, Bono e Thoresen (2003), em versão adaptada ao contexto brasileiro por Ferreira et al. (2013). Este instrumento é unifatorial, sendo composto por 12 itens (seis positivos e seis negativos), a serem respondidos em escala do tipo Likert de cinco pontos (1 - Discordo fortemente a 5 - Concordo fortemente). Exemplo de item: "Quando eu ten- to, geralmente sou bem sucedido". O índice de consistência interna (Alfa de Cronbach) no estudo de adaptação do instrumento (Ferreira et al., 2013) foi de 0,78 , e de 0,65 , no presente estudo.

O florescimento foi mensurado pela Escala de Florescimento de Diener et al. (2010), validada para o contexto brasileiro na presente pesquisa, mediante a análise fatorial confirmatória, por meio da modelagem de equações estruturais, no software R ( $R$ Core Team, 2017), no pacote Lavann (Rosseel, 2012). O instrumento é unifatorial, sendo composto de oito itens, a serem respondidos em escala do tipo Likert de sete pontos (1 - Discordo completamente a 7 - Concordo completamente). Os resultados obtidos (Qui-quadrado $=177,45(20)$, RMSEA $=0,10$, $\mathrm{CFI}=0,99$ e TLI $=0,98)$ confirmaram a estrutura unifatorial, com a retenção de todos os itens. Exemplo de item: "As pessoas me respeitam". O instrumento teve índice de consistência interna (Alfa de Cronbach) no estudo de Diener et al. (2010) de 0,87 e de 0,91, na atual investigação.

A saúde geral foi medida pelo Questionário de Saúde Geral de Goldberg (1972), adaptado ao contexto brasileiro por Gouveia et al. (2003). O instrumento é unifatorial (dimensão saúde geral), sendo composto por 12 itens, a serem respondidos em escala do tipo Likert de quatro pontos (1 - Absolutamente não a 4 - Muito mais do que de costume). Exemplo de item: "Tem sido capaz de enfrentar problemas?". A escala teve índice de consistência interna (Alfa de Cronbach) de 0,84 no estudo de validação ao contexto brasileiro (Gouveia et al., 2003) e, no presente trabalho, de 0,71 .

\section{Procedimentos de Coleta de Dados}

A coleta de dados foi realizada em versão online. Um formulário foi criado no aplicativo Google Docs, com os instrumentos utilizados. Os indivíduos foram convidados a participar por meio de mensagens em dois sites de relacionamento: o Facebook e o Linkedin, bem como pelo aplicativo Whatsapp, nos quais era enviado o link para acessar o questionário. Cumpre registar que, na presente pesquisa, todos os itens eram de preenchimento obrigatório. Assim, não houve missings, nem tão pouco, taxa de perda de dados. 


\section{Procedimentos de Análise de Dados}

$\mathrm{Na}$ análise dos dados, procedeu-se, inicialmente, à realização da Análise Fatorial Confirmatória da versão longa do Inventário Abrangente de Funcionamento Psicológico Positivo, por meio da Modelagem de Equações Estruturais, no software R ( $R$ Core Team, 2017), no pacote Lavann (Rosseel, 2012), como forma de se verificar sua estrutura. Uma vez que os dados não obedeceram ao pressuposto de normalidade (os itens do instrumento tiveram resultados significativos no teste de Shapiro-Wilk), utilizou-se o estimador weighted least square mean and variance ajusted (WLSMV), declarando-se os itens como variáveis categóricas-ordinais.

Em seguida, procedeu-se à seleção dos dez itens da versão breve de Su et al. (2014) para comporem a versão reduzida do instrumento. Posteriormente, essa versão foi submetida à Análise Fatorial Confirmatória, por meio da Modelagem de Equações Estruturais no software R (pacote Lavann), com estimador WLSMV, declarando-se os itens como variáveis categóricas-ordinais, a fim de se verificar a estrutura unifatorial desta versão.

Apoiando-se nas recomendações de Brown (2006), foram analisados os seguintes índices de ajuste: qui-quadrado (testa a probabilidade do modelo teórico se ajustar aos dados, sendo que quanto maior o valor do $\chi^{2}$ pior o ajustamento); Root-Mean-Square Error of Approximation (RMSEA - deve se situar abaixo de 0,08, aceitando-se valores até 0,10); Comparative Fit Index e Tucker-Lewis Index (CFI e TLI - são considerados aceitáveis valores acima de 0,90, preferivelmente acima de 0,95$)$. A consistência interna do instrumento foi calculada por meio do índice Alfa de Cronbach. Para a validade convergente do Inventário Abrangente de Funcionamento Psicológico Positivo, foram investigadas suas correlações (rho de Spearman) com os escores globais das medidas relacionadas ao instrumento em questão, nas quais foram consideradas como fracas as menores que 0,30 , como moderadas as de 0,30 a 0,70 , e como fortes, as acima de 0,70 (Barbetta, 2006).

\section{Procedimentos Éticos}

A pesquisa foi submetida ao Comitê de Ética em Pesquisa da instituição dos autores, com processo de número 013696/2017, sendo aprovada pelo CAE 65013017.7.0000.5289. Os respondentes manifestaram sua concordância em participar da pesquisa mediante o preenchimento do Termo de Consentimento Livre e Esclarecido.

\section{Resultados}

Com o intuito de avaliar a estrutura do Inventário Abrangente de Funcionamento Psicológico Positivo (IAFPP), foram testados três diferentes modelos, a partir da teoria de $\mathrm{Su}$ et al. (2014): (1) 18 fatores de primeira ordem (suporte, comunidade, confiança, respeito, solidão, pertencimento, engajamento, habilidades, aprendizagem, realização, autoeficácia, autoestima, controle, significado, otimismo, satisfação com a vida, afetos positivos e afetos negativos), que é a estrutura do instrumento original) $\left[\chi^{2}(g l)=\right.$ 2144,53 (1220); $\mathrm{CFI}=0,92$; TLI $=0,91$; RM$\mathrm{SEA}=0,03]$; (2) sete fatores de primeira ordem (relacionamento, engajamento, domínio, autonomia, significado, otimismo e bem-estar subjetivos) $\left[\chi^{2}(g l)=3445,61\right.$ (1356); CFI = 0,83; TLI $=0,82 ;$ RMSEA $=0,04]$, que são as dimensões globais do funcionamento psicológico positivo, segundo Su et al. (2014); (3) 18 fatores de primeira ordem com sete fatores de segunda ordem $\left[\chi^{2}(g l)=2699,51\right.$ (1342); CFI $=0,89 ;$ TLI $=$ $0,88$; $\operatorname{RMSEA}=0,04]$. Como pode ser observado nos resultados, o modelo de 18 fatores de primeira ordem foi o que apresentou os melhores índices de ajuste.

Os dados obtidos na análise fatorial confirmatória evidenciaram, portanto, que a estrutura do instrumento original foi replicada na presente pesquisa. Desse modo, a estrutura final do IAFPP, na amostra brasileira, ficou composta por 54 itens, distribuídos igualmente por 18 fatores correlacionados (Tabela 1). As médias das cargas fatoriais dos fatores variaram de 0,68 a 0,93 , enquanto os índices de consistência interna, calculados pelo Alfa de Cronbach, variaram de 0,70 a 0,95 (Tabela 2). 
Versões Breve e Abrangente.

Tabela 1

Fatores, Itens e Cargas Fatoriais Padronizadas do IAFPP

\begin{tabular}{lll}
\hline Fator & Itens & Cargas padroniz.
\end{tabular}

\begin{tabular}{|c|c|c|}
\hline & Há pessoas das quais posso depender para me ajudar & 0,47 \\
\hline \multirow[t]{3}{*}{ Suporte } & Há pessoas que me dão apoio e encorajamento & 0,80 \\
\hline & Há pessoas que gostam de mim como pessoa & 0,88 \\
\hline & Contribuo quando minha comunidade local precisa que algo seja feito & 0,77 \\
\hline \multirow[t]{3}{*}{ Comunidade } & Convido os meus vizinhos para minha casa & 0,68 \\
\hline & Procuro maneiras de ajudar os meus vizinhos quando eles estão em necessidade & 0,73 \\
\hline & Posso confiar nas pessoas da minha sociedade & 070 \\
\hline \multirow[t]{3}{*}{ Confiança } & Posso confiar nas pessoas do meu bairro & 0,65 \\
\hline & A maioria das pessoas que conheço são honestas & 0,70 \\
\hline & As pessoas me respeitam & 0,89 \\
\hline \multirow[t]{3}{*}{ Respeito } & As pessoas são educadas comigo &,- 76 \\
\hline & Sou tratado(a) com o mesmo respeito que os outros & 0,77 \\
\hline & Sinto-me solitário & 0,74 \\
\hline \multirow[t]{3}{*}{ Solidão } & Muitas vezes, sinto-me "de fora" & 0,86 \\
\hline & Não há ninguém de quem eu me sinta próximo & 0,66 \\
\hline & Sinto um sentimento de pertença à minha comunidade & 0,87 \\
\hline \multirow[t]{3}{*}{ Pertencimento } & Sinto um sentimento de pertença ao meu estado & 0,68 \\
\hline & Sinto um sentimento de pertença ao meu país & 0,72 \\
\hline & Fico totalmente absorvido (a) nas atividades que faço & 0,58 \\
\hline \multirow[t]{3}{*}{ Engajamento } & Na maioria das atividades que faço, eu me sinto energizado (a) & 0,85 \\
\hline & Fico excitado (a) quando eu trabalho em algo & 0,75 \\
\hline & Uso muito minhas habilidades na minha vida cotidiana & 0,84 \\
\hline \multirow[t]{3}{*}{ Habilidades } & Uso meus talentos frequentemente & 0,83 \\
\hline & Posso fazer aquilo no que sou bom todos os dias & 0,80 \\
\hline & Aprendi uma coisa nova ontem & 0,71 \\
\hline \multirow[t]{3}{*}{ Aprendizagem } & Aprender coisas novas é importante para mim & 0,63 \\
\hline & Aprendo sempre alguma coisa todos os dias & 0,76 \\
\hline & Estou alcançando a maioria dos meus objetivos & 0,88 \\
\hline \multirow[t]{3}{*}{ Realização } & Estou satisfazendo minhas ambições & 0,83 \\
\hline & Estou no caminho certo para alcançar meus sonhos & 0,81 \\
\hline & Posso ter sucesso se eu colocar minha mente em algo & 0,73 \\
\hline \multirow[t]{3}{*}{ Autoeficácia } & Estou confiante de que posso lidar com eventos inesperados & 0,77 \\
\hline & Acredito que sou capaz na maioria das coisas que faço & 0,76 \\
\hline & O que faço na vida é valioso e vale a pena & 0,86 \\
\hline \multirow[t]{3}{*}{ Autoestima } & As coisas que faço contribuem para a sociedade & 0,70 \\
\hline & O trabalho que faço é importante para outras pessoas & 0,72 \\
\hline & Outras pessoas decidem a maioria das minhas decisões de vida & 0,79 \\
\hline
\end{tabular}




\begin{tabular}{|c|c|c|}
\hline Fator & Itens & Cargas padroniz. \\
\hline \multirow[t]{3}{*}{ Controle } & As decisões de vida que tomo não são realmente minhas & 0,59 \\
\hline & Outras pessoas decidem o que eu posso ou não fazer & 0,92 \\
\hline & Minha vida tem um claro sentido de propósito & 0,85 \\
\hline \multirow[t]{3}{*}{ Significado } & Encontrei um significado satisfatório na vida & 0,87 \\
\hline & Sei o que dá sentido à minha vida & 0,85 \\
\hline & Sou otimista sobre meu futuro & 0,85 \\
\hline \multirow[t]{3}{*}{ Otimismo } & Tenho uma visão positiva da vida & 0,90 \\
\hline & Espero mais coisas boas na minha vida, do que ruins & 0,86 \\
\hline & De modo geral, minha vida está perto do meu ideal & 0,85 \\
\hline \multirow[t]{3}{*}{ Sat. com vida } & Estou satisfeito (a) com minha vida & 0,83 \\
\hline & Minha vida está indo bem & 0,88 \\
\hline & Sinto-me positivo (a) na maior parte do tempo & 0,94 \\
\hline \multirow[t]{3}{*}{ Afet positivos } & Sinto-me feliz na maior parte do tempo & 0,92 \\
\hline & Sinto-me bem na maior parte do tempo & 0,94 \\
\hline & Sinto-me negativo (a) na maior parte do tempo & 0,90 \\
\hline \multirow[t]{2}{*}{ Afet negativos } & Eu experimento sentimentos infelizes na maior parte do tempo & 0,79 \\
\hline & Sinto-me mal na maior parte do tempo & 0,85 \\
\hline
\end{tabular}

Tabela 2

Médias das Cargas Fatoriais e Índices de Consistência Interna dos Fatores de IAFPP

\begin{tabular}{|c|c|c|}
\hline Fatores & Médias Cargas Fatoriais & Consistência interna $(\alpha)$ \\
\hline Suporte & 0,72 & 0,73 \\
\hline Comunidade & 0,73 & 0,76 \\
\hline Confiança & 0,68 & 0,79 \\
\hline Respeito & 0,81 & 0,84 \\
\hline Solidão & 0,75 & 0,74 \\
\hline Pertencimento & 0,76 & 0,85 \\
\hline Engajamento & 0,73 & 0,76 \\
\hline Habilidades & 0,82 & 0,86 \\
\hline Aprendizagem & 0,70 & 0,70 \\
\hline Realização & 0,84 & 0,87 \\
\hline Autoeficácia & 0,75 & 0,78 \\
\hline Autoestima & 0,76 & 0,85 \\
\hline Controle & 0,77 & 0,78 \\
\hline Significado & 0,85 & 0,88 \\
\hline Otimismo & 0,87 & 0,90 \\
\hline Satisfação com a vida & 0,85 & 0,88 \\
\hline Afetos Positivos & 0,93 & 0,95 \\
\hline Afetos Negativos & 0,85 & 0,86 \\
\hline IBFPP & 0,68 & 0,90 \\
\hline
\end{tabular}


Posteriormente, foram selecionados os dez itens da versão breve de Su et al. (2014) para comporem a versão reduzida do instrumento (IBFPP). Tais itens pertenciam aos seguintes fatores: afetos positivos, otimismo, satisfação com a vida, significado, realização, autoestima, autoeficácia, engajamento, suporte e pertencimento. A análise fatorial confirmatória indicou que a es- trutura unifatorial do IBFPP apresentou índices adequados de ajuste: $\chi^{2}(g l)=91,90(35), \mathrm{CFI}=$ $0,97, \mathrm{TLI}=0,96$ e RMSEA $=0,05$. As cargas fatoriais dos dez itens variaram de 0,42 a 0,83 $(M=0,68)$ e o índice de consistência interna, calculado pelo Alfa de Cronbach, foi de 0,90. A Tabela 3 traz os itens e as cargas fatoriais padronizadas da versão final do IBFPP.

Tabela 3

Itens e Cargas Fatoriais Padronizadas do IBFPP

\begin{tabular}{lc}
\hline \multicolumn{1}{c}{ Itens } & Cargas padronizadas \\
\hline Minha vida tem um claro sentido de propósito & 0,42 \\
Sou otimista sobre meu futuro & 0,55 \\
Minha vida está indo bem & 0,67 \\
Sinto-me bem na maior parte do tempo & 0,78 \\
O que faço na vida é valioso e vale a pena & 0,60 \\
Posso ter sucesso se eu colocar minha mente em algo & 0,77 \\
Estou alcançando a maioria dos meus objetivos & 0,69 \\
Na maioria das atividades que faço, eu me sinto energizado (a) & 0,73 \\
Há pessoas que gostam de mim como uma pessoa & 0,78 \\
Sinto um sentimento de pertença à minha comunidade & 0,83 \\
\hline
\end{tabular}

Buscou-se avaliar, também, as evidências de validade convergente dos instrumentos. Para tanto, os escores obtidos em cada um dos instrumentos de coleta de dados foram computados a partir da média dos escores de seus respectivos itens, e as correlações entre essas escalas foram calculadas. Depois, foi testada a normalidade dos escores, por meio do Teste de Shapiro-Wilk. Como houve violação do pressuposto de normalidade, optou-se pelo uso da correlação de Spearman. Os dados obtidos (Tabela 4) evidenciaram que as correlações entre os fatores positivos do IAFPP, bem como do IBFPP, com as escalas de satisfação com a vida, otimismo, florescimento, avaliações autorreferentes e saúde geral, foram todas positivas, tendo variado de fracas a fortes $(0,27$ a 0,78$)$, com maioria, porém, variando de moderada a forte. Tais resultados confirmaram parcialmente a Hipótese 1. Verificou-se, ainda, que os fatores negativos do IAFPP apresentaram correlações negativas, de fracas a fortes $(-0,29$ a
- 0,60), com a satisfação com a vida, o otimismo, o florescimento, as avaliações autorreferentes e a saúde geral, com a maioria, porém, variando de moderada a forte, o que também confirmou parcialmente a Hipótese 2.

\section{Discussão}

O presente estudo teve como objetivo investigar as evidências iniciais de validade das versões abrangente e breve do Inventário de Funcionamento Psicológico Positivo, em amostras brasileiras. Em relação à análise fatorial confirmatória, os dados obtidos revelaram que o modelo de melhor ajuste da versão abrangente foi o de 18 fatores de primeira ordem. Assim, a versão final brasileira do Inventário Abrangente de Funcionamento Psicológico Positivo manteve os 54 itens do modelo original de Su et al. (2014). Ademais, os fatores da referida versão apresentaram bons índices de consistência interna. Tal 
Tabela 4

Correlações entre o IAFPP e IBFPP com Varáveis de Validade Convergente

\begin{tabular}{|c|c|c|c|c|c|}
\hline & Satisf Vida & Otimismo & Aval. Autorref. & Florescimento & Saúde Geral \\
\hline Suporte & $0,27^{*}$ & $0,28^{*}$ & $0,29^{*}$ & $0,32^{*}$ & $0,29^{*}$ \\
\hline Comunidade & $0,38^{*}$ & $0,37^{*}$ & $0,37^{*}$ & $0,53^{*}$ & $0,35^{*}$ \\
\hline Confiança & $0,42^{*}$ & $0,32^{*}$ & $0,36^{*}$ & $0,43^{*}$ & $0,37^{*}$ \\
\hline Respeito & $0,40^{*}$ & $0,38^{*}$ & $0,39^{*}$ & $0,44^{*}$ & $0,41^{*}$ \\
\hline Solidão & $-0,45^{*}$ & $-0,33^{*}$ & $-0,35^{*}$ & $-0,47^{*}$ & $-0,34^{*}$ \\
\hline Pertencimento & $0,39^{*}$ & $0,31^{*}$ & $0,36^{*}$ & $0,43^{*}$ & $0,39^{*}$ \\
\hline Engajamento & $0,51^{*}$ & $0,39^{*}$ & $0,45^{*}$ & $0,49^{*}$ & $0,41^{*}$ \\
\hline Habilidades & $0,54^{*}$ & $0,44^{*}$ & $0,43^{*}$ & $0,63^{*}$ & $0,45^{*}$ \\
\hline Aprendizagem & $0,47^{*}$ & $0,38^{*}$ & $0,43^{*}$ & $0,53^{*}$ & $0,40^{*}$ \\
\hline Realização & $0,69^{*}$ & $0,38^{*}$ & $0,47^{*}$ & $0,60^{*}$ & $0,44^{*}$ \\
\hline Autoeficácia & $0,52^{*}$ & $0,50^{*}$ & $0,52^{*}$ & $0,66^{*}$ & $0,44^{*}$ \\
\hline Autoestima & $0,45^{*}$ & $0,43^{*}$ & $0,47^{*}$ & $0,61^{*}$ & $0,42^{*}$ \\
\hline Controle & $-0,34^{*}$ & $-0,31^{*}$ & $-0,31^{*}$ & $-0,34^{*}$ & $-0,29^{*}$ \\
\hline Significado & $0,57^{*}$ & $0,46^{*}$ & $0,46^{*}$ & $0,63^{*}$ & $0,45^{*}$ \\
\hline Otimismo & $0,56^{*}$ & $0,57^{*}$ & $0,53^{*}$ & $0,69^{*}$ & $0,47^{*}$ \\
\hline Satisfação & $0,78^{*}$ & $0,43^{*}$ & $0,51^{*}$ & $0,68^{*}$ & $0,47^{*}$ \\
\hline Afet Pos & $0,67^{*}$ & $0,51^{*}$ & $0,54^{*}$ & $0,73^{*}$ & $0,50^{*}$ \\
\hline Afet Neg & $-0,56^{*}$ & $-0,49^{*}$ & $-0,48^{*}$ & $-0,60^{*}$ & $-0,49^{*}$ \\
\hline IAFPP & $0,68^{*}$ & $0,51^{*}$ & $0,55^{*}$ & $0,70^{*}$ & $0,51^{*}$ \\
\hline
\end{tabular}

$* p<0,01$.

resultado é semelhante ao encontrado no estudo de $\mathrm{Su}$ et al. (2014). Esses indicadores apontam para a possiblidade de estimar os escores latentes de maneira precisa (Cronbach, 1947).

A versão breve (IBFPP) também teve sua estrutura unifatorial confirmada, ficando composta pelos 10 itens que compunham a versão breve de Su et al. (2014). O resultado do índice de consistência interna (Alfa de Cronbach) do IBFPP mostra-se consistente com os achados de Su et al. (2014), bem como semelhante à investigação de Duan et al. (2016).

No que se refere à validade convergente das escalas, observou-se que os fatores positivos do IAFPP e o IBFPP correlacionaram-se de forma positiva com a satisfação com a vida, o otimismo, as avaliações autorreferentes, o florescimento e a saúde geral, enquanto que os fatores negativos apresentaram correlações negativas com estes construtos. Tais achados confirmaram parcialmente as Hipóteses 1 e 2. Eles se mostram, ainda, consistentes com os relatos de $\mathrm{Su}$ et al. (2014), que também encontraram correlações positivas dos fatores positivos do IAFPP e do IBFPP, com construtos correlatos ao bem- estar (satisfação com a vida, otimismo, avaliações autorreferentes e florescimento), bem como correlações negativas desses construtos com os fatores negativos do IAFPP. Tais resultados assemelham-se, ainda, ao estudo de Duan et al. (2016), no qual a versão breve correlacionou-se positivamente com a satisfação com a vida, o florescimento, o significado e propósito de vida e os afetos positivos.

Esses achados sugerem que as pessoas que vivenciam mais frequentemente estados positi- 
vos de funcionamento psicológico são também aquelas que se encontram mais satisfeitas com sua vida, mais otimistas em relação ao futuro, com melhor avaliação de si e florescimento. Por sua vez, os indivíduos que apresentam mais altos escores negativos na escala de funcionamento psicológico tendem a ser mais insatisfeitos com suas vidas e menos otimistas em relação ao futuro, além de reportarem maiores autoavaliações negativas e de não costumarem florescer. Uma vez que esses resultados corroboram a literatura (Duan et al., 2016; Su et al., 2014), há evidências, portanto, de validade convergente do IAFPP e do IBFPP com os construtos mencionados.

Tais achados podem ser vistos, ainda, como evidências de que o funcionamento psicológico positivo não pode ser considerado apenas como um indicador de felicidade ou satisfação, mas como um construto mais abrangente, que engloba aspectos relacionados à satisfação com a vida, às avaliações autorreferentes, ao otimismo e ao florescimento (Duan et al., 2016). Assim, pode-se afirmar que o Inventário de Funcionamento Psicológico Positivo avalia o bem-estar de forma mais abrangente e multidimensional, ao reunir indicadores de natureza mental, física e social.

No que tange às correlações encontradas com a saúde geral, os resultados são semelhantes aos encontrados por Su et al. (2014), que verificaram que ambas as escalas relacionam-se a resultados de saúde (saúde física, comportamentos saudáveis e qualidade de vida relacionada à saúde). Tais resultados permitem, assim, afirmar que os indivíduos que apresentam melhor nível de funcionamento psicológico positivo costumam apresentar melhores resultados de saúde, uma vez que uma concepção global e completa de saúde vai além da ausência de doença ou de sentimentos negativos (Su et al., 2014).

Os resultados do presente estudo indicam, assim, que os instrumentos IAFPP e o IBFPP apresentaram evidências adequadas de validade de estrutura interna, de precisão e de validade convergente. No entanto, cumpre registrar as limitações do estudo. Uma delas é que o instrumento utilizado foi de autorrelato e nem sempre o que é relatado pelos participantes condiz com a realidade. Outra limitação refere-se ao fato de que a maioria da amostra estava concentrada em uma única região do Brasil, o sudeste, e, em sua totalidade, possuía, pelo menos, o ensino médio. Assim, não é possível generalizar os resultados para as demais regiões brasileiras e, nem tampouco, para amostras de indivíduos sem ensino médio completo. Ademais, o presente estudo não explorou evidências de validade na predição de aspectos relacionados à saúde, conforme o estudo de Su et al. (2014). Desse modo, estudos futuros poderiam se dedicar a buscar evidências adicionais acerca da validade preditiva dos referidos instrumentos, mediante a mensuração de indicadores da saúde.

Sugere-se, ainda, a realização de investigações adicionais destinadas a aprofundar a rede nomológica do funcionamento psicológico positivo, em especial no que se refere a seu impacto em resultados de saúde e na qualidade de vida dos indivíduos. Estas pesquisas poderiam ser de natureza longitudinal, a fim de se obter maior compreensão acerca das relações entre essas variáveis. Novas pesquisas, em populações com outras características, como, por exemplo, trabalhadores em geral, com diferentes níveis de escolaridade, poderiam ser, também, realizadas. Em que pesem as limitações mencionadas, os achados ora obtidos permitem a conclusão de que as versões brasileiras do Inventário Abrangente de Funcionamento Psicológico Positivo e do Inventário Breve de Funcionamento Psicológico Positivo são apropriadas à mensuração do funcionamento mental, físico e social positivo do indivíduo, o que recomenda seu uso futuro em pesquisas desenvolvidas em uma variedade de contextos.

\section{Referências}

Albuquerque, A. S., \& Tróccoli, B. T. (2004). Desenvolvimento de uma Escala de Bem-Estar Subjetivo. Psicologia: Teoria e Pesquisa, 20(2), 153164. doi: 10.1590/S0102-37722004000200008

Bandeira, M., Bekou, V., Lott, K., Teixeira, M., \& Rocha, S. (2002). Validação transcultural do Teste de Orientação da Vida (TOV-R). Estudos de Psicologia (Natal), 7(2), 251-258. doi: 10.1590/S1413-294X2002000200006 
Barbetta, P. A. (2006). Estatística aplicada às ciências sociais. Florianópolis, SC: Editora da Universidade Federal de Santa Catarina.

Borsa, J. C., Damásio, B. F., \& Bandeira, D. R. (2012). Adaptação e validação de instrumentos psicológicos entre culturas: Algumas considerações. Paidéia (Ribeirão Preto), 22(53), 423-432. doi: 10.1590/S0103-863X2012000300014

Brown, T. A. (2006). Confirmatory factor analysis for applied research. New York: Guilford.

Coluci, M. Z. O., Alexandre, N. M. C., \& Milani, D. (2015). Construção de instrumentos de medida na área da saúde. Ciência e Saúde Coletiva, 20(3), 925-936. doi: 10.1590/141381232015203.04332013

Cronbach, J. L. (1947). Test "reliability": Its meaning and determination, Psychometrika, 22(1), 1-16. doi: http://dx.doi.org/10.1007/BF02289289

Diener, E., Emmon, R. A., Larsen, R. J., \& Griffin, S. (1985). The Satisfaction with Life Scale. Journal of Personality Assessment, 49(1), 71-75. doi: 10.1207/s15327752jpa4901_13

Diener, E., Oishi, S., \& Lucas, R. E. (2002). Subjective well-being: The science of happiness and life satisfaction. In C. R. Snyder \& S. J. Lopez (Eds.), Handbook of positive psychology (pp. 63-73). New York: Oxford University Press.

Diener, E., Wirtz, D., Biswas-Diener, R., Tov, W., Kim-Prieto, C., Choi, D., \& Oishi, S. (2009). New measures of well-being. In E. Diener (Ed.), Assessing well-being: The collected works of Ed Diener (pp. 247-266). Dordrecht, Netherlands: Springer.

Diener, E., Wirtz, D., Tov, W., Kim-Prieto, C., Choi, D.W., Oishi, S., \& Biswas-Diener, R. (2010). New well-being measures: Short scales to assess flourishing and positive and negative feelings. Social Indicators Research, 97(2), 143-156. doi: 10.1007/s11205-009-9493-y

Duan, W., Guan, Y., \& Gan, F. (2016). Brief Inventory of Thriving: A comprehensive measurement of well-being. Chinese Sociological Dialogue, 1(1), 15-31. doi: 10.1177/2397200916665230

Emmons, R. A. (1986). Personal strivings: An approach to personality and subjective well-being. Journal of Personality and Social Psychology, 51(5), 1058-1068. doi: 10.1037/00223514.51.5.1058

Ferreira, M. C., Thadeu, S. H., Masagão, V. C., Gottardo, L. F., Gabardo, L. M. D., Sousa, S. A. A.,
\& Mana, T. C. T. (2013). Escala de avaliações autorreferentes: Características psicométricas em amostras brasileiras. Avaliação Psicológica, 12(2), 227-232. Recuperado em http://www.redalyc.org/pdf/3350/335027505013.pdf

Goldberg, D. P. (1972). The detection of psychiatric illness by questionnaire: A technique for the identification and assessment of non-psychotic psychiatric illness. New York: Oxford University Press.

Gouveia, V. V., Barbosa, G. A., Andrade, E. O., \& Carneiro, M. B. (2005). Medindo a satisfação com a vida dos médicos no Brasil. Jornal Brasileiro de Psiquiatria, 54(4), 298-305. Recuperado em https:/www.researchgate.net/ publication/279194945_Medindo_a_satisfacao_ com a vida dos medicos no Brasil

Gouveia, V. V., Chaves, S. S., Oliveira, I. C. P., Dias, M. R., Gouveia, R. S. V., \& Andrade, P. R. (2003). A utilização do QSG-12 na população geral: Estudo de sua validade de construto. Psicologia: Teoria e Pesquisa, 19(3), 241-248. doi: 10.1590/S0102-37722003000300006

Huta, V., \& Ryan, R. M. (2010). Pursuing pleasure or virtue: The differential and overlapping well-being benefits of hedonic and eudaimonic motives. Journal of Happiness Studies, 11(6), 735-762. doi: 10.1007/ s10902-009-9171-4

Judge, T. A., \& Bono, J. E. (2001). A rose by any other name: Are self-worth, generalized self-efficacy, neuroticism, and locus of control indicators of a common construct? In B. W. Roberts \& R. Hogan (Eds.), Personality psychology in the workplace: Decade of behavior (pp. 93-118). Washington, DC: American Psychological Association.

Judge, T. A., Erez, A., Bono, J. E., \& Thoresen, C. J. (2003). The core self-evaluations scale: Development of a measure. Personnel Psychology, 56(2), 303-331. doi: 10.1111/j.1744-6570.2003. tb00152.x

Judge, T. A., Locke, E. A., \& Durham, C. C. (1997). The dispositional causes of job satisfaction: A core evaluations approach. Research in Organizational Behavior, 19(1), 151-188. Retrieved from https://www.researchgate.net/publication/2280 79159_The_dispositional_causes_of job_satisfaction_A_core_evaluations_approach

Keyes, C. L. M. (2002). The mental health continuum: From languishing to flourishing in life. Journal of Health and Social Behavior, 43(2), 
207-222. Retrieved from https://www.n cbi.nlm. nih.gov/pubmed/12096700

Machado, W. L., Bandeira, D. R., \& Pawlowski, J. (2013). Validação da Psychological Well-being Scale em uma amostra de estudantes universitários. Avaliação Psicológica, 12(2), 263-272. Recuperado em http://pepsic.bvsalud. org/scielo.php?script $=$ sci_arttext\&p id $=$ S167704712013000200017

Mendonça, H., Caetano, A., Ferreira, M. C., Sousa, I. F., \& Silva, A. J. (2014). Florescimento e afetos no trabalho. In M. M. M. Siqueira (Ed.), Novas medidas de comportamento organizacional: Ferramentas de diagnóstico e de gestão (pp. 172-177). Porto Alegre, RS: Artmed.

R Core Team. (2017). R: A language and environment for statistical computing. Vienna, Austria: $\mathrm{R}$ Foundation for Statitiscal Computing. Retrieved from http://www.R-project.org/

Rosseel, Y. (2012). Lavaan: An R package for structural equation modeling. Journal of Statistical Software, 48(2), 1-36. Retrieved from http:// www.jstatsoft.org/v48/i02/paper

Ryan, R. M., \& Deci, E. L. (2008). Self-determination theory and the role of basic psychological needs in personality and the organization of behavior. In O. P. John, R. W. Robbins, \& L. A. Pervin (Eds.), Handbook of Personality: Theory and Research (pp. 654-678). New York: The Guilford Press.

Ryff, C. D. (1989). Happiness is everything, or is it? Explorations on the meaning of psychological well-being. Journal of Personality and Social Psychology, 57(6), 1069-1081. doi: 10.1037/0022-3514.57.6.1069

Ryff, C. D., \& Essex, M. J. (1992). The interpretation of life experience and well-being: The sample case of relocation. Psychology and Aging, 7(2), 507-517. Retrieved from https://www.ncbi.nlm. nih.gov/pubmed/1466819

Ryff, C. D., \& Keyes, C. L. M. (1995). The structure of psychological well-being revisited. Journal of Personality and Social Psychology, 69(4), 719. 727. Retrieved from http://midus.wisc.edu/findings/pdfs/830.pdf
Sarriera, J. C., Schwarcz, C., \& Câmara, S. (1996). Bem-estar psicológico: Análise fatorial da escala de Goldberg (GHQ-12) numa amostra de jovens. Psicologia: Reflexão e Crítica, 9(2), 323-336. Recuperado em https://www.scienceopen.com/document?vid=623d4037-c1a8-401c-b2be-c0afd237b8b0

Scheier, M. F., \& Carver, C. S. (1985). Optimism, coping, and health: Assessment and implication of generalized outcome expectancies. Health Psychology, 4(3), 219-247. Retrieved from https://www.ncbi.nlm.nih.gov/pubmed/4029106

Seligman, M. E. P. (1995). The optimistic child: A proven program to safeguard children against depression and build lifelong resilience. New York: Harper Perennial.

Seligman, M. E. P. (2004). Felicidade autêntica: Usando a nova psicologia positiva para a realização permanente. Rio de Janeiro, RJ: Objetiva.

Seligman, M. E. P. (2011). Flourish: A visionary new understanding of happiness and well-being. New York: Free Press.

Seligman, M. E. P., \& Csikszentmihalyi, M. (2000). Positive psychology: An introduction. American Psychologist, 55(1), 5-14. doi: 10.1037/0003066X.55.1.5

Snyder, C. R., \& Lopez, S. J. (2009). Psicologia positiva: Uma abordagem científica e prática das qualidades humanas (R. C. Costa, Trad.). Porto Alegre, RS: Artmed.

Su, R., Tay, L., \& Diener, E. (2014). The development and validation of the Comprehensive Inventory of Thriving (CIT) and the Brief Inventory of Thriving (BIT). Applied Psychology: Health and Well Being, 6(3), 251-279. doi: 10.1111/aphw.12027

Zanon, C., Bastianello, M. R., Pacico, J. C., \& Hutz, C. S. (2013). Desenvolvimento e validação de uma escala de afetos positivos e negativos. Psico-USF, 18(2), 193-201. doi: 10.1590/S141382712013000200003

Recebido: 03/04/2017

$1^{a}$ revisão: $14 / 04 / 2017$

$2^{a}$ revisão: $30 / 06 / 2017$

$2^{a}$ revisão: 05/09/2017

Aceite final: 05/09/2017

(cc) BY 1 (c) O(s) autor(es), 2018. Acesso aberto. Este artigo está distribuído nos termos da Licença Internacional Creative Commons Atribuição 4.0 (http://creativecommons.org/licenses/by/4.0/), que permite o uso, distribuição e reprodução sem restrições em qualquer meio, desde que você dê crédito apropriado ao(s) autor(es) original(ais) e à fonte, fornecer um link para a licença Creative Commons e indicar se as alterações foram feitas. 